\title{
Análise, Projeto, Desenvolvimento e Avaliação de Jogos Sérios e Afins: uma revisão de desafios e oportunidades
}

\author{
Rafaela Vilela da Rocha $^{1}$, Ig Ibert Bittencourt ${ }^{2}$, Seiji Isotani ${ }^{1}$ \\ ${ }^{1}$ Instituto de Ciências Matemáticas e de Computação - Universidade de São Paulo \\ (USP) - São Carlos - SP - Brasil \\ ${ }^{2}$ Instituto de Computação - Universidade Federal de Alagoas (UFAL) \\ Maceió - AL - Brasil
}

\begin{abstract}
For serious games can be successfully used for educational and training purposes, they should be analyzed, designed, developed and evaluated in a systematic and holistic way, i.e., considering their development lifecycle and the requirements of their parties and interrelations. However, these development phases, requirements and challenges in the area are spread across several articles and books available in the literature. This article aims at compiling and presenting the research findings on the serious games life cycle and seven major challenges. We believe that the review of the literature and discussions presented in this work can contribute to give directions about this field of research, its challenges and opportunities.
\end{abstract}

Resumo. Para que os jogos sérios possam ser utilizados com sucesso, para fins educacionais e de treinamento, eles devem ser analisados, projetados, desenvolvidos e avaliados de forma sistemática e holística, i.e., considerando seu ciclo de vida de desenvolvimento e a abrangência dos requisitos de suas partes e inter-relações. Entretanto, estas fases de desenvolvimento, os requisitos e desafios na área estão distribuídos em diversos artigos e livros disponíveis na literatura. Este artigo apresenta os resultados da revisão da literatura sobre o desenvolvimento de jogos sérios e áreas afins, e discute sete principais desafios. Esta revisão pode contribuir para direcionar pesquisas futuras nesta área, seus desafios e oportunidades.

\section{Introdução}

Jogos sérios, em inglês serious games, são jogos utilizados com propósito de ensinoaprendizagem ou treinamento e não apenas de entretenimento [Aldrich, 2005; BNDES, 2014a]. Eles envolvem o uso de tecnologias de jogos digitais com o propósito de simular problemas do mundo real. Suas aplicações têm sido eficientes nas áreas de saúde, defesa, negócios, turismo, entre outras [Aldrich, 2005; Mattar, 2010; Novak, 2010]. Eles são essenciais particularmente em atividades que envolvem risco à vida, ao patrimônio e ao meio ambiente, pois reduz os riscos reais e custos dos treinamentos práticos tradicionais [Green III, 2000; Phelan, 2008]. Entretanto, seu desenvolvimento é um processo complexo e custoso, devido a necessidade de integração de diferentes áreas de conhecimentos (aprendizagem, avaliação, simulação e de jogos em si, além do conteúdo no domínio de aplicação), e isto requer profissionais qualificados, com o uso de ferramentas e processos próprios [Balci, 2012; EMS, 2006]. 
Para oferecer suporte para sua produção, diferentes metodologias emergiram, além das existentes nas áreas de design de jogos, modelagem de simulação e design instrucional. As metodologias que são focadas nestas áreas específicas enfatizam aspectos do produto (jogo, simulação ou material instrucional) que tem diferenças significativas em relação a um jogo sério. Materiais educacionais são lineares; têm o conteúdo estruturado de forma lógica para auxiliar o instrutor; com ênfase no processo em vez de foco no aprendizado e aprendizes [Gordon e Zemke, 2000]. Por outro lado, jogos em geral são não lineares, possuem regras, estratégias, desafios, recompensas, níveis e feedback contínuo, porém focam apenas no entretenimento e diversão quando não são produzidos para fins educacionais [Michael e Chen, 2006; Novak, 2010]. Ao passo que simulações também são não lineares, mas focam na representação física e comportamental do sistema real que está sendo modelado [Goldsman, 2007]. Assim, um dos desafios em desenvolver um jogo sério é balancear e integrar os requisitos do jogo, simulação e conteúdo [Engström et al., 2011; Hays, 2005].

As metodologias que unificam alguns elementos comuns a estas áreas, fazem isto em processos paralelos e não abrangem todos os requisitos necessários, criando uma lacuna entre os domínios de conhecimentos [Adams et al. 2007; Akili e Cagiltay, 2006; Aslan e Balci, 2015; 2015; Becker e Parker, 2012; Freitas e Jarvis, 2006; Hays, 2005; Kirkley, Tomblin e Kirkley, 2005; Rodrigues, Machado e Valença, 2010; Van Der Zee, Holkenborgb e Robinson, 2012; Zin, Jaafar e Yue, 2009].

Neste contexto, este artigo discute os sete principais desafios que devem ser abordados durante o ciclo de vida de desenvolvimento de jogos sérios (incluindo a análise, projeto, desenvolvimento e avaliação) (na seção 2). Na seção 3, é apresentado o estado da arte das metodologias existentes e suas limitações. Na seção 4, os resultados são analisados e discutidos, seguidos das considerações finais (na seção 5).

\section{Ciclo de Vida dos Jogos Sérios: desafios e oportunidades}

Os desafios na produção de jogos sérios podem ser agrupados em desafios relacionados aos jogos sérios em si (produto final, Figura 1, itens 1 e 2), ao seu desenvolvimento (Figura 1, itens 3, 4 e 5), ou aos ambos (jogo sério e metodologia, Figura 1, itens 6 e 7).

O primeiro desafio está nas características e requisitos do produto a ser criado: software de jogo e simulação, objeto de aprendizagem e treinamento, instrumento de avaliação, que requerem a abrangência das áreas de treinamento e avaliação, domínio de aplicação, além de simulação e jogo. Um jogo sério é um software com interface gráfica (com informações e interações possíveis), porém deve ter elementos fundamentais que garantem o sucesso dos jogos, tais como, regras, estratégias, desafios, recompensas, níveis e feedback contínuo [Novak, 2010; Domingues, 2011], bem como ter fidelidade física, comportamental e psicológica do que está sendo simulado [Feinstein e Cannon, 2002; Goldsman, 2007]. Jogos sérios precisam atender aos princípios de aprendizagem efetiva, que geralmente os jogos contemplam (aprendizagem ativa, prática das competências, motivações, objetivos e pré-requisitos claros, vários cenários ou problemas) [Trybus, 2014], porém de forma a balancear jogabilidade com o conteúdo de aprendizagem [Engström et al., 2011]. Além disto, eles precisam medir, avaliar os resultados e fornecer feedback para os aprendizes. Isso inclui verificar o grau em que os aprendizes aplicam as competências requeridas, analisar e determinar as causas do bom e mau desempenho, incluindo o processo e não apenas o resultado [Salas e Rosen, 2007, 
ABNT, 2001; Mattar, 2010; Salas e Cannon-Bowers, 2001; Maciuszek, Weicht e Martens, 2012]. Além de que, o feedback deve ser imediato, para corrigir ou reforçar o bom resultado durante todo o processo de treinamento, e não somente no final (resultado do jogo) [Salas et al., 2009]. O desafio é atender e balancear todos estes requisitos do produto final.

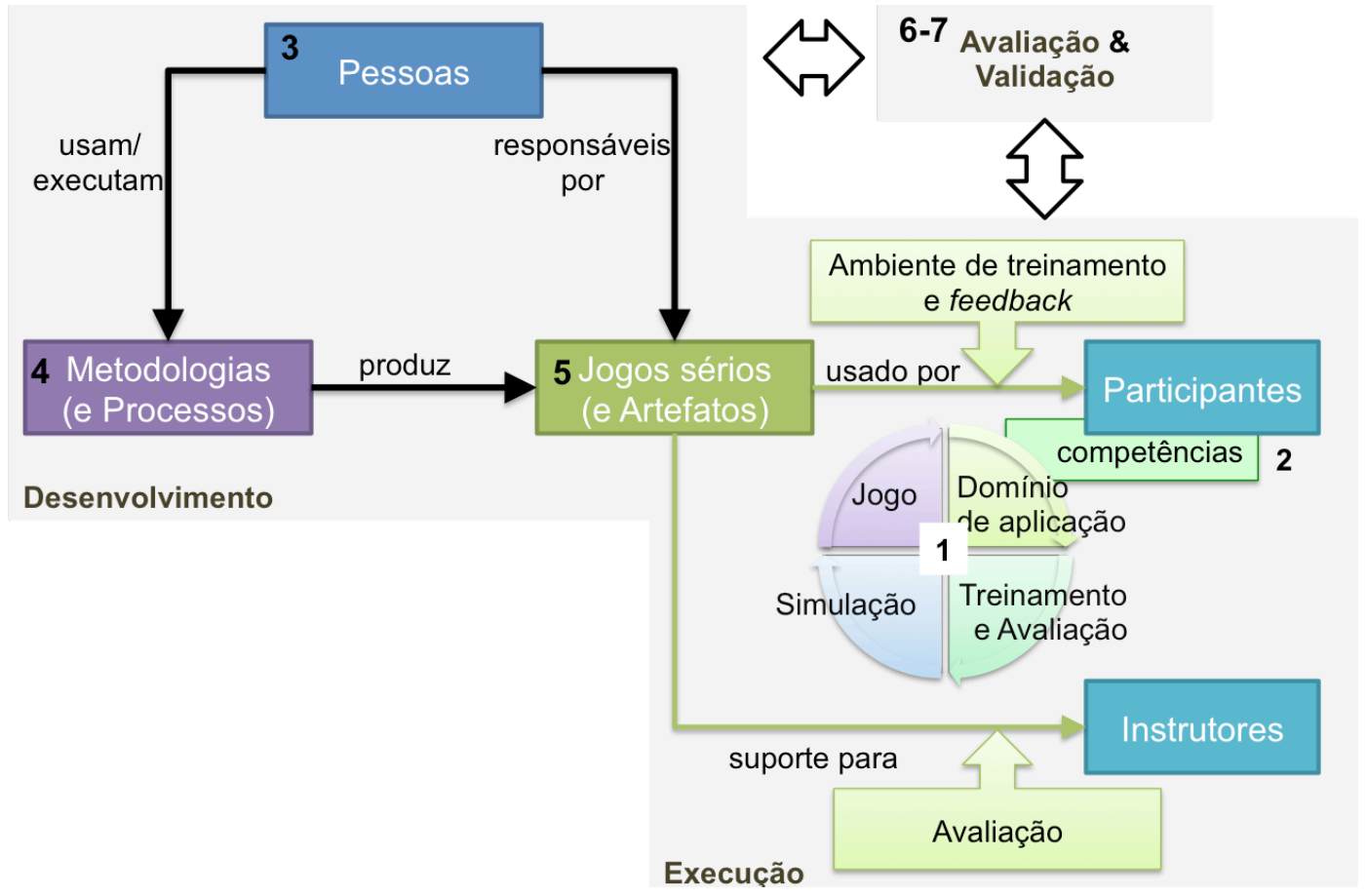

Figura 1. Produção de jogos sérios e seus desafios [adaptado de Rocha, 2014]

O segundo desafio é relacionado ao treinamento dentro do domínio de aplicação. É requisito saber quem são os aprendizes e quais competências devem ser treinadas. As competências são formadas por conhecimentos, habilidades e atitudes pessoais empregadas no desempenho de uma determinada atividade. O conhecimento é ter informações (saber o quê e o porquê); a habilidade é ter a técnica e capacidade de aplicar o conhecimento (saber como fazer); a atitude é o querer fazer (motivação, vontade e comprometimento) [Abbad et al., 2012; Boog, 2001]. Os conhecimentos são aprendidos; as habilidades podem ser exercitadas e treinadas; ao passo que, as atitudes se formam com o resultado de experiências por meio de feedbacks de reforço ou correção. O desafio está em criar atividades ao longo dos níveis do jogo, com objetivos claros e desafiantes, que exijam o uso destas competências requeridas.

O terceiro desafio é relacionado à integração dos conhecimentos e atividades dos profissionais envolvidos, pois desenvolver um treinamento requer conhecimento específico e uma equipe multidisciplinar para planejar e implementar o jogo sério. A equipe técnica é formada de um ou mais especialistas no domínio (profissionais na área operacional, tática ou estratégica da organização, pedagogos, professores, psicólogos). A equipe de desenvolvedores é formada por um ou mais especialistas em modelagem e simulação, analistas, designers, programadores, modeladores $3 \mathrm{D}$, gerentes de projeto, entre outros [Balci, 2012; Chandler, 2012; Kelly et al., 2007; Pace, 2004; Rankin et al., 2011]. Cada um desses atores tem diferentes visões (para o quê?) e missões (o quê? e como?) do treinamento que será criado, bem como diferentes responsabilidades, metas e atividades durante o processo de desenvolvimento [Balci, 2012; Chandler, 2012; Novak, 
2010; Rankin et al., 2011]. O desafio é integrar as atividades destes diferentes atores, em processos colaborativos e cooperativos.

O quarto desafio é a qualidade da metodologia de criação do jogo, que é influenciada por 4 P's: Processos (usados para criar os artefatos); Produtos (artefatos); Projeto (características de planejamento, gerenciamento de risco, controle e monitoramento); e Pessoas (qualidade das pessoas envolvidas na criação de artefatos) [Balci, 2012]. A qualidade das pessoas depende de suas competências no assunto, ao passo que a qualidade do projeto depende de planejamento, controle e avaliação durante todo o processo [Campos e Guimarães, 2008]. Por outro lado, produtos e processos dependem de "o que" e "como" se quer produzir. A definição e padronização de processos e modelos de produtos aumenta a probabilidade de qualidade do jogo. Outro fator que pode contribuir é a visão sistêmica das pessoas envolvidas no planejamento e monitoramento dos processos e do projeto.

O quinto desafio é reusar e estender os jogos sérios e seus artefatos. De modo geral, suas aplicações são específicas e são dependentes da arquitetura de suporte. Dessa forma, as alterações na aplicação implicam em alterações nessa arquitetura, o que é complexo e custoso [Oliveira, Crowcroft e Slater, 2003]. Assim, como requisitos não funcionais, a arquitetura e artefatos devem ser flexíveis, possibilitar reuso, customização, integração e interoperabilidade [EMS, 2006; Strassburger, Schulze e Fujimoto, 2008]. Além dos artefatos de software, também é importante viabilizar o reuso dos artefatos de planejamento e projeto de desenvolvimento [Balci, 2012]. Isto pode ser alcançado por meio da definição e padronização de projetos, processos e atividades, o que reflete positivamente na qualidade de sua produção.

O sexto desafio é referente a integração da avaliação nos diferentes pontos de vista das áreas afins de desenvolvimento de jogos sérios. A avaliação do desempenho humano é apenas uma das avaliações necessárias e possíveis [ABNT, 2001; Boyle, Connolly, Hainey, 2011; Salas et al., 2009]. As avaliações podem ter diferentes objetivos, serem realizadas em diferentes momentos e fornecer feedbacks para diferentes pessoas envolvidas, por exemplo, avaliações de simulações interativas, de jogos sérios, da aprendizagem, do programa de treinamento, do impacto na organização, etc. [Abbad et al., 2012; ABNT, 2001; Kirkpatrick e Kirkpatrick, 2006]. Desta forma, é essencial definir "o que" e "como" será avaliado e "quando" cada uma ocorre.

O último desafio é referente às validações dos jogos sérios desenvolvido para seus usos intencionados no domínio real: simular, treinar e avaliar. As validações visam garantir que o que está sendo desenvolvido representam e simulam o mundo real. Entretanto, da mesma forma que nas avaliações, as validações podem ter diferentes propósitos: validar o modelo de simulação, o jogo sério como instrumento de aprendizagem e avaliação; o software ou a interface, etc. [Balci, 2010; Feinstein e Cannon, 2002; Roza et al., 2010; Roza, Voogd e Sebalj, 2012; Sargent, 2010]. Além disto, verificações podem ser um requisito para algum tipo de validação, bem como as próprias avaliações. Deste modo, é essencial definir "o que" e "como" será validado e em que momento acontecerá, de modo a garantir sua eficácia para o uso intencionado.

\section{Estado da Arte}

Uma revisão da literatura foi realizada para obter uma compreensão geral do estado da arte e as limitações das metodologias existentes. Foram pesquisados artigos nos 
principais motores de busca, em congressos e periódicos das áreas relacionadas; e livros e sites de referência que descrevem metodologias de desenvolvimento de jogos sérios e produtos relacionados; além de consultadas as referências citadas nesses trabalhos. Elas podem ser agrupadas em metodologias de: (1) Design de jogos de entretenimento [Chandler, 2012; Godoy, 2010; Laubisch e Clua, 2010; Novak, 2010; Schuytema, 2008]; (2) Modelagem de simulações [Balci, 2012; Banks et al., 2001; IEEE, 2003 e 2010; Ford, 2004]; (3) Design de instruções e treinamentos [ABNT, 2001; Dick, Carey e Carey, 2004]; (4) Design de simulações e jogos educacionais [Akilli e Cagiltay, 2006; Adams et al, 2007; Aslan e Balci, 2015; Hays, 2005; Zin, Jaafar e Yue, 2009]; e (5) Desenvolvimento de jogos sérios [Becker e Parker, 2012; Freitas e Jarvis, 2006; Kirkley, Tomblin e Kirkley, 2005; Rodrigues, Machado e Valença, 2010; Van Der Zee, Holkenborgb e Robinson, 2012].

As metodologias foram avaliadas em grupos, a descrição e análise completa é apresentada em [Rocha, 2014], nos seguintes itens: (1) autor e proposta, (2) área de aplicação, (3) design do jogo, (4) modelagem da simulação (dividida em distribuída, lógica e física), (5) verificação e validação, (6) planejamento do aprendizado e seu conteúdo, (7) planejamento do programa de treinamento e das competências treinadas, (8) avaliação do desempenho do aprendiz, (9) avaliação do artefato criado (jogo, simulação, treinamento, etc.), e (10) limitações. Um resumo das principais características é apresentado no Quadro 1, as limitações são descritas a seguir.

Quadro 1. Avaliação das metodologias de desenvolvimento de jogos sérios e áreas afins [adaptado de Rocha, 2014]

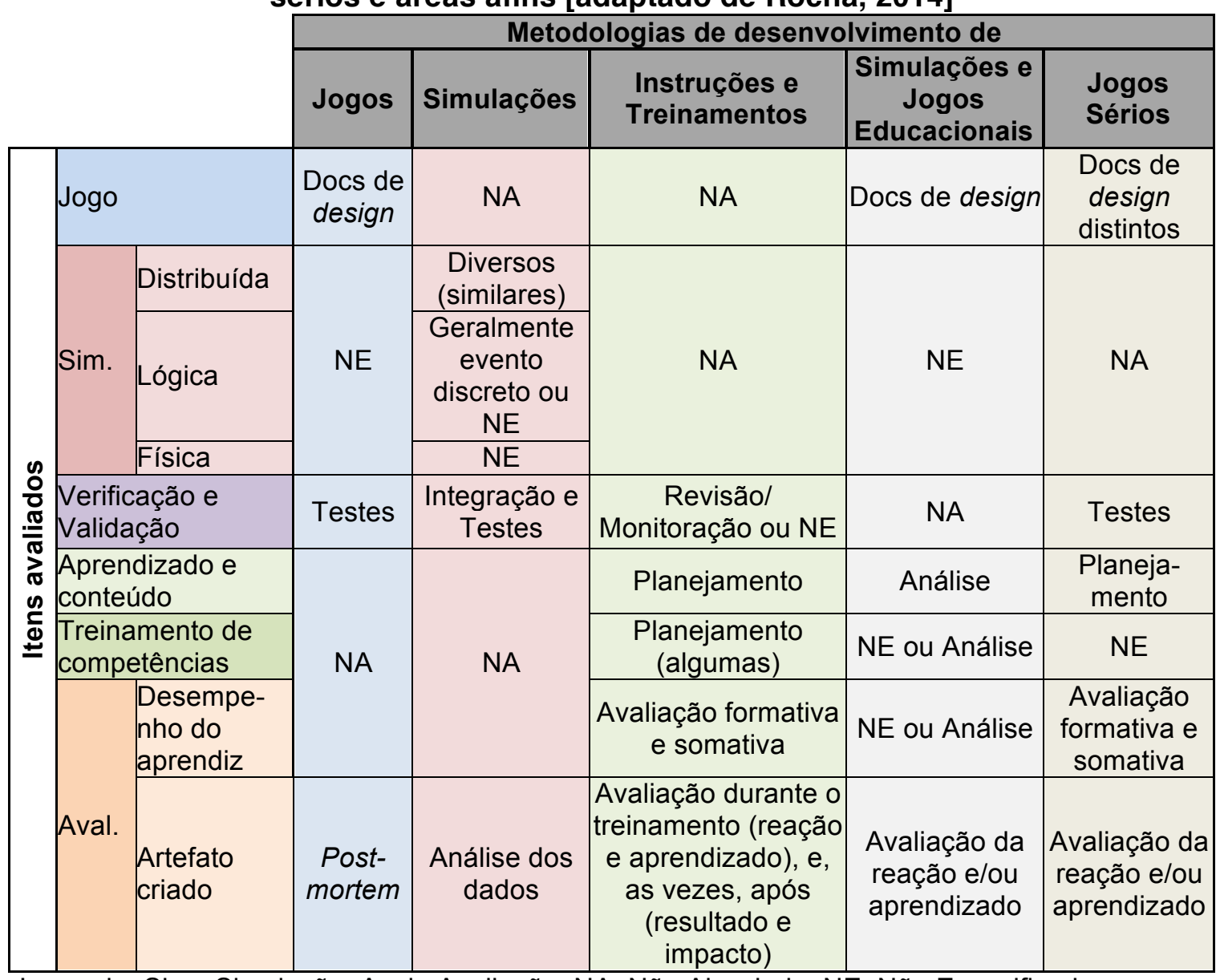

Legenda: Sim.: Simulação; Aval.: Avaliação; NA: Não Abordado; NE: Não Especificado 
De forma geral, as limitações das metodologias de desenvolvimento de jogos são: (1) Faltam detalhamentos e documentações dos processos e dos artefatos produzidos; e (2) Faltam inclusões de requisitos de simulação, aprendizagem, treinamento e avaliação. Ao passo que, as metodologias de modelagem de simulações: faltam detalhamentos dos requisitos instrucionais, de treinamento, de avaliação e do modelo de representação. Por outro lado, as especificações de design instrucionais e de treinamentos: (1) Não abrangem requisitos de: jogos e simulações; e (2) Há diferenças significativas entre materiais educacionais e jogos sérios, por ex., linearidade, conteúdos estruturados e lógicos para auxiliar o instrutor, ênfase no processo em vez de foco no aprendizado e aprendizes.

Em relação as metodologias de criação de simulações e jogos educacionais, elas não oferecem apoio para avaliação e feedback do desempenho do aprendiz. Ao passo que, as metodologias de desenvolvimento de jogos sérios: têm foco no design instrucional e do jogo, porém sem integração detalhada na metodologia. Além disto, elas não apoiam a avaliação do jogo sério.

\section{Análise e Discussões}

De forma geral, estas metodologias não descrevem o ciclo de vida completo compreendendo os múltiplos requisitos do jogos sérios para treinamento e avaliação. Muitas metodologias são focadas em áreas específicas e apenas enfatizam elementos de jogo, ou da simulação, ou da instrução/treinamento. Outras unificam alguns elementos destas áreas, mas sem abranger todos os requisitos necessários [Becker e Parker, 2012; Freitas e Jarvis, 2006; Kirkley, Tomblin e Kirkley, 2005; Van Der Zee, Holkenborgb e Robinson, 2012].

Uma das barreiras para adoção de jogos sérios em ambientes corporativos ou educacionais, é eles não terem definições dos objetivos de aprendizagem e das competências a serem treinadas [Donovan, 2012]. Isto é um reflexo da deficiência da integração e do equilíbrio das áreas relacionadas durante o desenvolvimento de um novo produto, i.e. jogo sério, que tem características próprias (referente ao primeiro desafio apresentado na seção 2). Nesse sentido, também é importante verificar que tipo de jogo pode ser usado para cada tipo de necessidade e alinhar os diferentes tipos e estratégias de jogos (simulação, ação, quebra cabeças, etc.) aos distintos tipos e objetivos de aprendizagem (por exemplo, habilidades motoras, habilidades intelectuais, atitudes, conhecimento, etc.) e de avaliação (segundo e sexto desafios). Este alinhamento deve ser realizado a partir da análise das necessidades de cada instituição, com o uso de normas e procedimentos padronizados, e em conjunto com teorias, tais como, de aprendizagem, instrucionais, de design instrucional, de desempenho humano, e de avaliação [ABNT, 2001; Bloom, 1956; Kolb e Kolb, 2005; Salas et al. 2009; Kirkpatrick e Kirkpatrick, 2006].

Em relação as equipes que participam da produção (terceiro desafio), as equipes de design instrucional, que criam produtos educacionais, geralmente contêm apenas designers instrucionais e especialistas no domínio, ao passo que as equipes desenvolvedoras de jogos nem sempre possuem designers instrucionais ou especialistas de treinamento [Quinn, 2005; BNDES, 2014b]. Entretanto, a solução não é apenas contratar estes profissionais para produzirem um jogo sério, é necessário sinergia e distribuição das tarefas entre as equipes durante sua produção (quarto desafio) [Moraes 
et al., 2012]. As soluções existentes seguem duas linhas distintas: ou propõem o desenvolvimento em paralelo de design instrucional e de design do jogo, ou integram os designs em um mesmo processo, porém em atividades e artefatos distintos [Kirkley, Tomblin e Kirkley, 2005; Nadolski et al., 2008; Rodrigues, Machado e Valença, 2010; Zin, Jaafar e Yue, 2009]. Porém, a dificuldade ou falta de comunicação entre essas equipes pode gerar atrasos ou até mesmo retrabalho.

Além de não haver uma integração efetiva nas atividades realizadas, os artefatos produzidos também não são integrados, cada um desenvolve seus próprios produtos (quinto desafio). Além disso, é necessário também desenvolver novos modelos que integrem os pontos fortes das áreas educacionais (com teorias de aprendizagem e desempenho humano, métodos de ensino, etc.) e de jogos; a partir de uma análise das vantagens e deficiências de cada área; além da inclusão de outras áreas, tais como, psicologia e gerenciamento de projetos [Eck, 2007; Rocha, 2014].

Ainda em relação a produção do jogo sério, quarto e quinto desafios, as metodologias não oferecem um desenvolvimento holístico e integrado durante todo o ciclo de vida [Taylor et al., 2013]. Muitas vezes, são apenas utilizados processos de desenvolvimento de software tradicional que criam uma aplicação fortemente acoplada com arquitetura, sem se preocupar com questões de reuso em novas aplicações, e interoperabilidade e extensão das existentes [Oliveira, Crowcroft e Slater, 2003].

Além disso, é muito importante também realizar atividades de verificação e validação de todos os recursos criados ao longo do desenvolvimento, as quais muitas metodologias não abordam (sétimo desafio). Isto deve incluir tanto as verificações e validações do modelo de simulação, referentes a validade representacional e comportamental [Balci, 2011; Roza et al., 2010; Roza, Voogd e Sebalj, 2012; Sargent, 2010], quanto a validação do jogo sério como instrumento educacional de treinamento e avaliação [Feinstein e Cannon, 2002].

\section{Considerações Finais}

Neste artigo foram apresentados e discutidos desafios e oportunidades de pesquisa na produção e uso de jogos sérios como instrumentos de aprendizagem, treinamento e avaliação. Esta área de pesquisa vem atraindo e integrando pesquisadores de diversas áreas da Ciência da Computação, Educação e Psicologia. Em específico, foram descritos sete desafios: produto final; competências requeridas; integração de diferentes profissionais; sistematização e padronização de artefatos e processos; reuso e extensão de artefatos; avaliação; e validação.

As pesquisas em áreas tradicionais e mais bem desenvolvidas, tais como, Engenharia de Software, Design Instrucional, Interface Homem-Computador, Modelagem e Simulação, Educação, e Psicologia, podem beneficiar e produzir melhores soluções na área de jogos sérios. Para isto, é necessário a integração destas pesquisas, tanto do ponto de vista de metodologia (pessoas, processos, produtos, projetos), quanto em relação ao desenvolvimento, uso e validação de jogos sérios (existentes ou novos). Entretanto, há uma lacuna entre estas áreas que oferecem apoio à criação de jogos sérios, e não há metodologia que integre todos os requisitos e supere os desafios citados.

Estas limitações são, muitas vezes, causadas pelo próprio contexto de pesquisa, em que jogos sérios são inseridos em outras áreas, tais como, soluções em simulações 
distribuídas, engenharia de software, realidade virtual e aumentada, treinamento e desenvolvimento humano, sistemas de medição de desempenho humano; ou em subáreas de pesquisa, tais como, desenvolvimento de jogos para dispositivos específicos (por exemplo, consoles ou celulares) ou jogos para domínios particulares (por exemplo, saúde, educação, defesa, negócios).

Como trabalhos futuros, uma nova metodologia iterativa e integradora, criada a partir dos desafios descritos, deverá ser validada por meio de estudos de casos com equipes multidisciplinares; e os jogos sérios produzidos deverão ser validados junto aos especialistas do domínio e usuários finais (validade representacional e educacional).

\section{Agradecimentos}

Agradecemos ao CNPq e CAPES (projeto INCT-Sec, processos 237216/2012-4 e 150613/2015-6) pelo apoio financeiro.

\section{Referências}

ABBAD, G.S.; et al. (2012). "Medidas de Avaliação em Treinamento, Desenvolvimento e Educação: ferramentas para gestão de pessoas". Porto Alegre: Artmed.

ABNT. (2001). "NBR ISO 10015:2001 - Gestão da Qualidade: diretrizes para treinamento". Rio de Janeiro: ABNT.

ADAMS, W.K.; et al. (2007). A Study of Educational Simulations Part I - Engagement and Learning. Journal of Interactive Learning Research, v. 12. p. 397-419.

AKILLI, G.K.; CAGILTAY, K. (2006). "An Instructional Design/Development Model for the Creation of Game-Like Learning Environments: the FIDGE model”. In: Pivec, M. (Ed.), Affective and Emotional Aspects of Human-Computer Interaction: Game-Based and Innovative Learning. IOS Press, p. 93-112.

ALDRICH, C. (2005). "Learning by doing: a comprehensive guide to simulations, computer games, and pedagogy in e-learning and other educational experiences". San Francisco: Pfeiffer.

ASLAN, S.; BALCI, O. (2015). GAMED: Digital Educational Game Development Methodology. Simulation, v. 91, n. 4, p. 307-319.

BALCI, O. (2010). Golden Rules of Verification, Validation, Testing, and Certification of Modeling and Simulation Applications. SCS M\&S Magazine, Oct. 2010, n. 4.

BALCI, O. (2012). A Life Cycle for Modeling and Simulation. Simulation, v. 88, n. 7. p. 870883.

BANKS, J.; et al. (2001). "Discrete-Event System Simulation”. New Jersey: Prentice-Hall.

BECKER, K.; PARKER, J. (2012). "Serious Instructional Design: ID for digital simulations and games". In: P. RESTA (ED.), Proceedings of Society for Information Technology \& Teacher Education International Conference. p. 2480-2485.

BLOOM, B.S. (1956). "Taxonomy of Educational Objectives: the classification of educational goals - Handbook I: Cognitive Domain". New York: McKay.

BNDES. (2014a). "Banco Nacional de Desenvolvimento Econômico e Social. Mapeamento da Industria Brasileira e Global de Jogos Digitais". Relatório Final.

BNDES. (2014b). "I Censo da Indústria Brasileira de Jogos Digitais". Relatório Final.

BOOG, G.G. (2001). "Manual de Treinamento e Desenvolvimento: um guia de operação". São Paulo: MAKRON Books.

BOYLE, E.; CONNOLLY, T.M.; HAINEY, T. (2011). The role of psychology in understanding the impact of computer games. Entertainment Computing, v. 2, n. 2. p. 69-74.

BYERS, C. (2010). "Combining Instructional Design and Game Design". In: Gaming and Simulation: concepts, methodologies, tools and applications, v. I, Hershey: IGI Global. p. 
CBIE-LACLO 2015

Anais do XXVI Simpósio Brasileiro de Informática na Educação (SBIE 2015)

359-372.

CAMPOS, J.P.; GUIMARÃES, J.P. (2008). "Em busca da eficácia em treinamento: norma ABNT-NBR-ISO-10015:2001 - gestão da qualidade - diretrizes para treinamento".

CHANDLER, H.M. (2012). "Manual de Produção de Jogos Digitais". 2a. Ed. Porto Alegre: Bookman.

DICK, W.; CAREY, L. CAREY, J.O. (2004). "The Systematic Design of Instruction”. 6th ed. Allyn \& Bacon.

DOMINGUES, D.G. (2011). Protótipos para a criação de jogos digitais: aplicações no ensino de design de games. Tese (Doutorado em Design) - Depto de Artes e Design, PUC do Rio de Janeiro.

DONOVAN, L. (2012). "The Use of Serious Games in the Corporate Sector: a state of the art report". Learnovate Centre.

ECK, R.V. (2007). "Building Artificially Intelligent Learning Games". In: GIBSON, D.; ALDRICH, C.; PRENSKY, M. (Eds.) Games and Simulations in Online Learning: research $\&$ development frameworks. Hershey, PA, USA: Idea Group, p. 271-307.

EMS. (2006). "Essentials of Modeling \& Simulation". Disponível em: http://nmso.navy.mil/ems/welcome.html. Acesso em: fev. 2011.

ENGSTRÖM, H.; et al. (2011). Making a Game of the Old Testament Balancing Authenticity, Education and Entertainment. IADIS International Journal on WWW/Internet, v. 9, n. 1.

FEINSTEIN, A. H.; CANNON, H. M. (2002). Constructs of Simulation Evaluation. Simulation \& Gaming, v. 33, n. 4. p. 425-440.

FORD, F. (2004). The Euclid RTP 11.13 SE Development \& Exploitation Process (SEDEP). European Simulation Interoperability Workshops, 04E-SIW-037, p. 1-10.

FREITAS, S.; JARVIS, S. (2006). A Framework for Developing Serious Games to meet Learner Need. I/ITSEC. p. 1-11.

GODOY, A.; BARBOSA, E.F. (2010). "Game-scrum: an approach to agile game development". In: Simpósio Brasileiro de Jogos e Entretenimento Digital. p. 292-295.

GOLDSMAN, D. (2007). "Introduction to Simulation". In: Winter Simulation Conference, Washington, DC. Piscataway: IEEE Press. p. 26-37.

GORDON, J., ZEMKE, R. (2000). The Attack on ISD. Training Magazine, v. 37, n. 4. p. 42 .

GREEN III, W. G. (2000). "Exercise Alternatives for Training Emergency Management Command Center Staffs". Command Center Staffs: Universal Publishers.

HAYS, R.T. (2005). "The Effectiveness of Instructional Games: a literature review and discussion”. Naval Air Warfare Center Training Systems Division (Technical Report 2005 $004)$.

IEEE. (2003). "1516.3-2003- IEEE Recommended Practice for High Level Architecture (HLA): federation development and execution process (FEDEP)". New York: IEEE.

IEEE. (2010). “1730-2010- Distributed Simulation Engineering and Execution Process (DSEEP)". Washington: IEEE.

KELLY, H.; et al. (2007). "How to Build Serious Games". In: Communications of the ACM. v. 50, n. 7. p. 45-49.

KIRKLEY, S.; TOMBLIN, S.; KIRKLEY, J. (2005). Instructional Design Authoring Support for the Development of Serious Games and Mixed Reality Training. I/ITSEC.p. 1-11.

KIRKPATRICK, D.L.; KIRKPATRICK, J.D. (2006). "Evaluating Training Programs: The Four Levels". 3rd Edition. San Francisco: Berrett-Koehler.

LAUBISCH, A.; CLUA, E. (2010). "Scrum4Games: Uma aplicação do Scrum para projetos de games focada em game design”. In: Simpósio Brasileiro de Jogos e Entretenimento Digital, p. $178-187$.

MACIUSZEK, M.; WEICHT, M.; MARTENS, A. (2012). "Seamless Integration of Game and Learning Using Modeling and Simulation". In: Winter Simulation Conference. p. 16111620 . 
CBIE-LACLO 2015

Anais do XXVI Simpósio Brasileiro de Informática na Educação (SBIE 2015)

MATTAR, J. (2010). "Games em Educação: como os nativos digitais aprendem”. São Paulo: Pearson Prentice Hall.

MICHAEL, D.; CHEN, S. (2006). "Serious Games: games that educate, train, and inform". Boston: Thomson Course Technology.

NADOLSKI, R. J. et al. (2008). Serious Games for Higher Education: a framework for reducing design complexity. Journal of Computer Assisted Learning, v. 24, n. 5. p. 420-432.

NOVAK, J. (2010). "Desenvolvimento de Games". São Paulo: Cengage.

OLIVEIRA, M.; CROWCROFT, J.; SLATER, M. (2003). An Innovative Design Approach to Build Virtual Environments Systems. Workshop on Virtual Environments. p. 143-151.

PACE, D.K. (2004). "Modeling and simulation verification and validation challenges". Johns Hopkins APL Technical Digest, v. 25, n. 2. p. 163-172.

PHELAN, T.D. (2008). "Emergency Management and Tactical Response Operations: bridging the gap". Burlington: Elsevier.

QUINN, C. N. (2005). "Engaging Learning: designing e-learning simulation games". San Francisco: Pfeiffer.

ROCHA, R.V. (2014). Metodologia iterativa e modelos integradores para desenvolvimento de jogos sérios de treinamento e avaliação de desempenho humano. Tese (Doutorado em Ciência da Computação) - Departamento de Computação, Universidade de São Carlos, São Carlos, SP.

RODRIGUES, H.F.; MACHADO, L.S., VALENÇA, A.M. (2010). "Definição e Aplicação de um Modelo de Processo para o Desenvolvimento de Serious Games na Área de Saúde". In: Proc. Congresso da Sociedade Brasileira de Computação - Workshop de Informática Médica. p. 1532-1541.

ROZA, M.; et al. (2010). Generic Methodology for Verification and Validation for Training Simulations. I/ITSEC. p. 1-12.

ROZA, M.; VOOGD, J.; SEBALJ, D. (2012). The Generic Methodology for Verification and Validation to support acceptance of models, simulations and data. JDMS. v. 10 n. 4. p. 347365.

SALAS, E.; CANNON-BOWERS, J. A. (2001). The Science of Training: a decade of progress. Annual Review of Psychology, v. 52. p. 471-499.

SALAS, E.; et al. (2009). Performance Measurement in Simulation-Based Training: A Review and Best Practices. Simulation Gaming, v. 40, n. 3. p. 328-376.

SALAS, E.; ROSEN, M.A. (2007). "Best Practices for Performance Measurement in Military Simulation-based Training: observations from the field". Report, University of Central Florida. p. 1-29.

SARGENT, R.G. (2010). "Verification and Validation of Simulation Models". In: Winter Simulation Conference. p. 166-183.

SCHUYTEMA, P. (2008). "Design de Games: uma abordagem prática”. São Paulo: Cengage Learning.

STRASSBURGER, S.; SCHULZE, T.; FUJIMOTO, R. M. (2008). "Future Trends in Distributed Simulation and Distributed Virtual Environments". Peer Study Final Report. 48p.

TAYLOR, S.J.E.; et al. (2013). Grand Challenges in Modeling and Simulation: expanding our horizons. SIG on Simulation. p. 409-414.

TRYBUS, J. (2010). "Game-Based Learning: What it is, Why it Works, and Where it's Going". NMI White Paper. New Media Institute, New York.

VAN DER ZEE, D.J.; HOLKENBORGB, B.; ROBINSON, S. (2012). Conceptual modeling for simulation-based serious gaming. Decision Support Systems, v. 54. p. 33-45.

ZIN, N.A.M; JAAFAR, A.; YUE, W.S. (2009). Digital Game-Based Learning (DGBL) Model And Development Methodology For Teaching History. WSEAS Transactions On Computers, v.2, n.8. p. 322-333. 